\title{
Might Gene Duplication and Neofunctionalization Contribute to the Sexual Lability Observed in Fish?
}

\author{
Jonika Edgecombe $^{\mathrm{a}}$ Lara Urban $^{\mathrm{a}} \quad$ Erica V. Todd $^{\mathrm{b}}$ Neil J. Gemmell ${ }^{\mathrm{a}}$ \\ aDepartment of Anatomy, University of Otago, Dunedin, New Zealand; 'bShool of Life and Environmental Sciences, \\ Deakin University, Queenscliff, VIC, Australia
}

\section{Keywords}

Sex determination $\cdot$ Sexual differentiation $\cdot$ Sex reversal .

Sequential hermaphrodite · Gene duplication ·

Neofunctionalization - Developmental plasticity · Genetics ·

Epigenetics · Comparative genomics

\begin{abstract}
Sex determination and differentiation varies widely across vertebrates, but is most dramatically diverse in fishes. Among fishes sex reversal and sex change are observed in 41 teleost families spanning 7 orders. These sex-changing fish perhaps highlight better than any other system that sex determination is not the narrow and fixed construct we once thought, but a plastic trait that is better viewed as a reaction norm. However, while this stunning transformation is increasingly understood, a fundamental question arises, which is why some fish species have retained this inherent plasticity in sexual fate, while others have not? Here, we explore our current understanding of sex change in fish, some of the factors that permit and constrain sex reversal, and posit that gene duplication and neofunctionalization contribute to the sexual lability observed in fish.

(c) 2021 S. Karger AG, Basel
\end{abstract}

\section{Introduction}

Sex determination and differentiation varies widely across vertebrates [Bachtrog et al., 2014; Capel, 2017; Ortega-Recalde et al., 2020] (Fig. 1). Sex determination is the process during embryonic development that triggers the bipotential primordia to make the fate decision to become testes or ovaries [Devlin and Nagahama, 2002]. Sexual differentiation follows this; the bipotential gonad differentiates and secondary sex characteristics develop [Devlin and Nagahama, 2002]. Genetic sex determination can be governed by single genes of large effect or by the interactions of multiple genes [Bachtrog et al., 2014; Capel, 2017]. Further, some species undergo environmental sex determination where environmental conditions such as temperature affect sexual fate [Bachtrog et al., 2014; Capel, 2017]. Other systems include haplodiploidy, hermaphroditism or the integration of many signals towards sex determination (as observed in zebrafish) [Bachtrog et al., 2014; Capel, 2017; Ortega-Recalde et al., 2020]. Sexual differentiation can also be influenced by hormone sensitivity and environmental changes [Capel, 2017; Ortega-Recalde et al., 2020] (Fig. 1). The sexual fate is not always fixed, with sex reversal and sex change oc-

Correspondence to:

Neil J. Gemmell, neil.gemmell@otago.ac.nz 


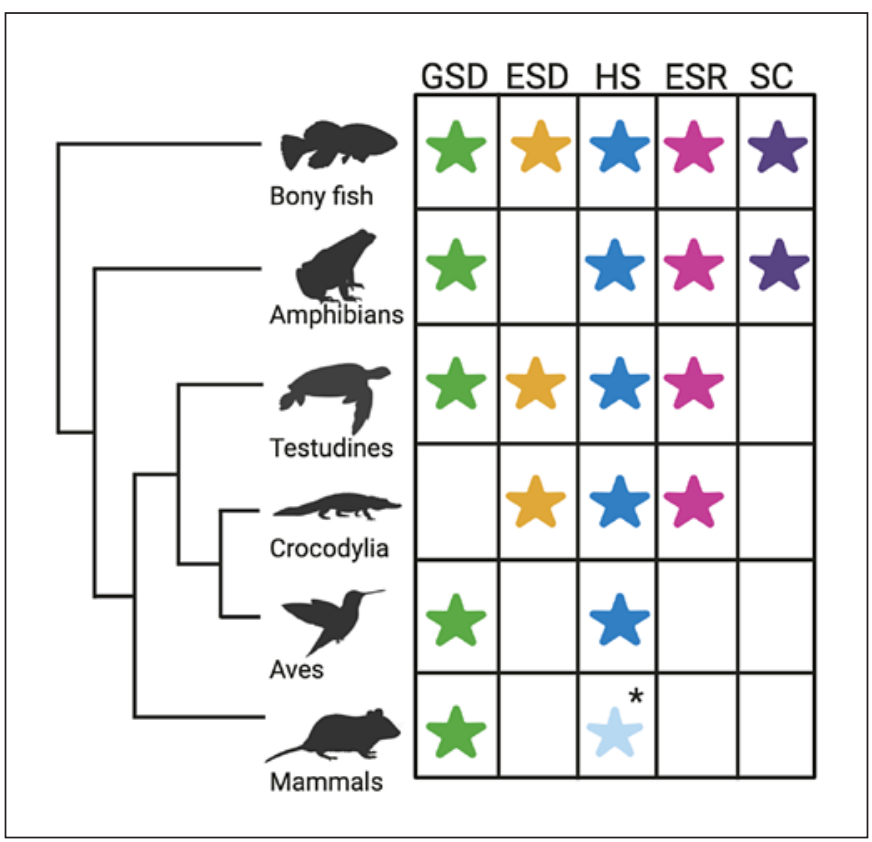

Fig. 1. Vertebrate sex determination and sex change. GSD, genetic sex determination; ESD, environmental sex determination; HS, hormone sensitivity; ESR, environmental sex reversal; SC, sex change. ${ }^{*}$ Hormone sensitivity does not affect sex determination in eutherians but does in metatherians [Ortega-Recalde et al., 2020]. Phylogenetic tree is not to scale. Figure adapted from Ortega-Recalde et al. [2020] and Capel [2017]. Created with BioRender.com.

curring in some species, most notably within teleost fish. As such, sex determination is not the narrow and fixed construct that is often assumed by the human world, but rather, sex determination can occur in many different ways; sex is a plastic trait that is better viewed as a reaction norm [Ah-King and Nylin, 2010; Todd et al., 2016].

\section{Fish and Hermaphroditism}

In vertebrates, hermaphroditism is largely restricted to teleost fish [Bachtrog et al., 2014; Todd et al., 2016]. This is in contrast to gonochoristic systems where the sexes are separate [Policansky, 1982]. Sequential hermaphrodites go through at least one breeding season functionally as one sex, and then go through subsequent breeding seasons as another sex [Warner, 1975; Policansky, 1982; Munday et al., 2006]. This key distinction differentiates sequential hermaphrodites from (1) simultaneous hermaphrodites who have functional ovaries and testes at the same time, and (2) gonochorists, such as zebrafish, which may go through a period of transient hermaphroditism where all juveniles produce immature oocytes before gonads develop fully as either ovaries or testes [Takahashi, 1977].

Sequential hermaphroditism can occur as a transformation from female to male (protogynous), male to female (protandrous), or serially in both directions (bidirectional) [Warner, 1975; Policansky, 1982; Nakamura et al., 2005; Munday et al., 2006]. Sex change can occur as part of the usual life history (e.g., in groupers) [Nakamura et al., 2005] or in response to social and visual cues or environmental changes (e.g., in wrasse) [Warner and Swearer, 1991]. The initiation of sex change is complex, involving many levels of integration across the brain and gonads, and the specific mechanisms remain to be elucidated [Lamm et al., 2015].

Hermaphroditism is spread widely across the teleost branch, occurring in 41 teleost families across 7 orders [Kuwamura et al., 2020]. Why hermaphroditism is so common in teleosts is unknown, but one intriguing possibility is that their anatomy and genomes predispose them towards this sexual flexibility [Adolfi et al., 2019]. Teleosts lost the müllerian duct during evolution and have therefore developed structures for gamete release that are completely independent from the urinary system, creating optimal anatomic and developmental preconditions for physiological sex change [Adolfi et al., 2019]. Teleosts also present unique genetic features including whole-genome duplication (WGD) and molecular adaptations in sex associated genes, such as $a m h / a m h r 2$, that may have increased the potential to sex change in adult life [Adolfi et al., 2019]. However, phylogenetic reconstructions [Mank et al., 2006] have revealed no teleost orders with exclusive hermaphroditism. As hermaphroditism is embedded within clades where gonochorism is dominant, sequential hermaphroditism has presumably evolved independently multiple times in the evolution of teleost fish.

\section{Why Change Sex?}

As sex is integral to reproductive success and directly impacts fitness, the marked variation in sex determination and differentiation processes across teleost fish is somewhat unexpected - particularly given that other key developmental processes such as body axis specification are highly conserved [Capel, 2017]. This leads to the question: What is the advantage of changing sex? Sex change requires extensive remodelling, and thus requires significant energy and resources. The dominant theory explain- 
ing sequential hermaphroditism is the size advantage model (SAM) [Ghiselin, 1969]. This model proposes that if it is advantageous to be bigger as one sex, it may be favourable for an animal to be sequentially hermaphroditic so that lifetime reproductive success is optimised - particularly if size has negligible impact on the other sex (or smaller size is favoured in the other sex). Recent evidence has shown a tight correlation between mating systems and sex allocation, consistent with the SAM in the Labridae [Hodge et al., 2020]. As a result, the SAM has been extended to include the various size-specific ways in which sex change could increase reproductive success, such as positive correlation between size and female fecundity (in favour of protandry), mating systems (mate monopolization by dominant males in favour of protogyny), and differential growth rates [Warner, 1988; Hodge et al., 2020]. There remains contention over whether the SAM is sufficient to explain bidirectional sex change. In this scenario, the risk of movement model [Ghiselin, 1969] proposes that in a low-density population where habitats are isolated and predation risk of movement is high, it is advantageous to be able to become either male or female such that any 2 individuals can form a reproductive pair [Ghiselin, 1969; Nakashima et al., 1996; Munday, 2002].

\section{Who Changes Sex and How?}

Given sex change can provide clear fitness advantages, the obvious question is why sex change is not more widespread? Likely the answer is quite simply that many species are constrained due to genomic, developmental or life history factors.

Sex change occurs across 41 families and 7 orders in the teleost families [Kuwamura et al., 2020]. Sparidae, Serranidae, Labridae, Pomacentridae, and Gobiidae are families in which the mechanisms of sequential hermaphroditism have been investigated in detail. In many serranids, sex change is inevitable, e.g., protogynous Epinephelus merra (honeycomb grouper) undergo sex change after about 5 years [Nakamura et al., 2005]. Similarly, the protandrous Acanthopagrus schlegelii (black porgy) are functional males for the first 2 years of life before $30-50 \%$ of them undergo sex change as part of their life course [Wu et al., 2010]. Anatomical and histological analyses have demonstrated black porgy to have a bisexual gonad, which functions as a male gonad for the first 2 spawning seasons. In sex-changing individuals, the testicular tissue subsequently regresses and the ovarian tissue dominates, whereas in non-sex-changing individuals the ovarian tissue regresses [Huang et al., 2002; Lee et al., 2008].

In the Labridae family, Thalassoma bifasciatum (bluehead wrasse) is protogynous, and sex change is induced based on social cues. Bluehead wrasse have a haremic social structure where a terminal-phase (TP) male is dominant, monopolizing its harem of females. If the TP male dies/is removed, often it is the largest female who undergoes sex change and takes over the dominant TP male's role [Warner and Swearer, 1991]. This is in line with the finding that protogynous sex change in Labridae is correlated with mating behaviour such as mate monopolization where male size advantage is beneficial [Hodge et al., 2020]. Anatomically, protogynous wrasse gonads undergo a complete restructure; the ovary completely transforms into a testis [Nakamura et al., 2005].

In the Pomacentridae family, protandrous anemonefish (genus Amphiprion) also experience sex change under social influence [Fricke and Fricke, 1977; Hattori, 2005]. However, most Amphiprion species are monogamous, and mate selection is limited by ecological factors - social group size is limited by the size of the host anemones [Hattori, 2005]. Protandrous anemonefish adult males have a bisexual gonad comprising mature testicular tissue and immature ovarian tissue. When sex change occurs, the testicular tissue regresses, and the ovarian tissue matures [Nakamura et al., 2005]. No protandrous males are observed to have only male tissue [Reinboth, 1988], indicating that juveniles develop ovaries first.

Members of the Gobiidae family are similarly limited as a sessile species. However, gobiids change sex bidirectionally, which appears to provide an advantage through being able to always secure a heterosexual breeding pair. When same-sex pairs are created, sex change occurs in approximately 5 or 10 days for protogynous and protandrous change, respectively [Nakamura et al., 2005]. Anatomically, in the bidirectional gobiid Trimma okinawae species, individuals possess ovaries and testes simultaneously: functionally female fish develop ovaries and immature testes, and vice versa [Kobayashi et al., 2005].

\section{Master Regulators and Effectors of Sex Determination}

Across vertebrates, and especially teleost fish, sex determination pathways involve a common set of effector genes, but the master regulator or sex determination genes are highly variable. Both mammalian and teleost models of sex determination and differentiation include 


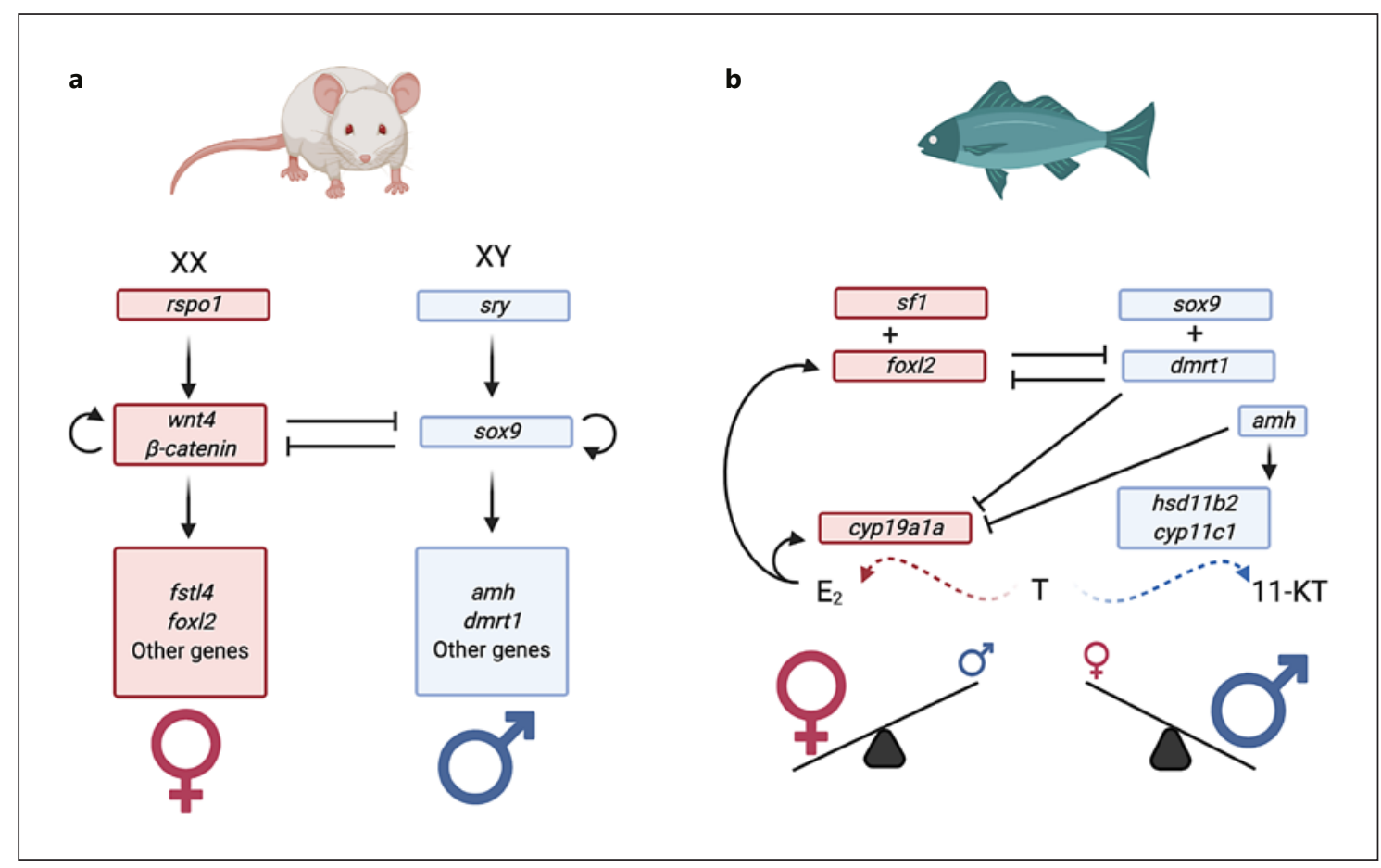

Fig. 2. Models of sex determination and development. a Mammalian model of sex determination. b Teleost sexual development pathway. Adapted from Todd et al. [2016, 2019]. Created with BioRender.com.

common effectors within male (e.g., sox9, amh) and female (e.g., foxl2, wnt4) antagonistic pathways that battle for dominance to maintain gonadal fate [Herpin and Schartl, 2011] (Fig. 2). In most mammals, there is a known master regulator (SRY gene on the Y chromosome) that triggers sex determination, whereas the master regulators identified in fish are diverse and unknown for most species. Master regulators in fish include amhr2 in Takifugu rubripes [Kamiya et al., 2012], amhy in Odontesthes hatcheri [Hattori et al., 2012], a tandem duplicate of amhy in Oreochromis niloticus [Li et al., 2015] or $s d Y$ in Oncorhynchus mykiss [Yano et al., 2012]. In the medaka clade, multiple genes have taken on the role as the sex-determining factor [Matsuda, 2018]. $d m y$ (aka $d m r t 1 b$ ) is the sex-determining gene for Oryzias latipes [Matsuda et al., 2002] and Oryzias curvinotus [Matsuda, 2018], gsdfY fulfils this role for Oryzias luzonesis [Myosho et al., 2012], and sox3Y is the master sex gene for Oryzias duncena [Matsuda, 2018]. While sex is determined by a single master regulator in only a few fish species, the core genetic mechanisms of sex determination are strongly maintained across teleosts, but vary in how the network upstream of sex steroid synthesis is initiated and in the regulatory loops that maintain one fate and repress the other (Fig. 2).

Gene Duplication, Neofunctionalization, and the Sexual Plasticity of Fish
Among the key players in the vertebrate sex determination and sexual development pathways, a few genes continually come to the fore in the literature on fish sex determination and differentiation. These include but are not limited to cyp19a1a, cyp19a1b, hsd11b2, sox9, foxl2, amh, dmrt1, wnt4, and rspo1 [Lamm et al., 2015; Liu et al., 2015; Todd et al., 2016, 2019; Zhang et al, 2019; Ortega-Recalde et al., 2020]. As shown in Figure 2, there are canonical "female" and "male" genes. The aromatase enzyme that converts testosterone to oestrogen in the gonad is cyp19ala, while cyp19a1b fulfils this role in the brain [Chiang et al., 2001; Page et al., 2010; Zhang et al., 2014]. Gonadal aromatase cyp19a1a is associated with ovarian development, function, and maintenance. Its product, $17 \beta$-estradiol (E2), forms a positive feedback loop on its transcriptional regulators sf1 (steroidogenic factor 1, which has multiple roles in the early bipotential gonad and in steroidogenesis for both sexes) [Herpin and Schartl, 2015] and foxl2 (forkhead box L2) [Guiguen et al., 2010; Todd et al., 2016]. foxl2 also negatively regulates $d m r t 1$ (double-sex and mab-3 related transcription factor) which is a canonically "male" gene [Todd et al., 2016]. 11ß-hydroxy-steroid dehydrogenase $(h s d 11 b 2)$ and $11 ß$-hydroxylase (cyp11c1) are also steroidogenesis enzymes involved in the synthesis of 


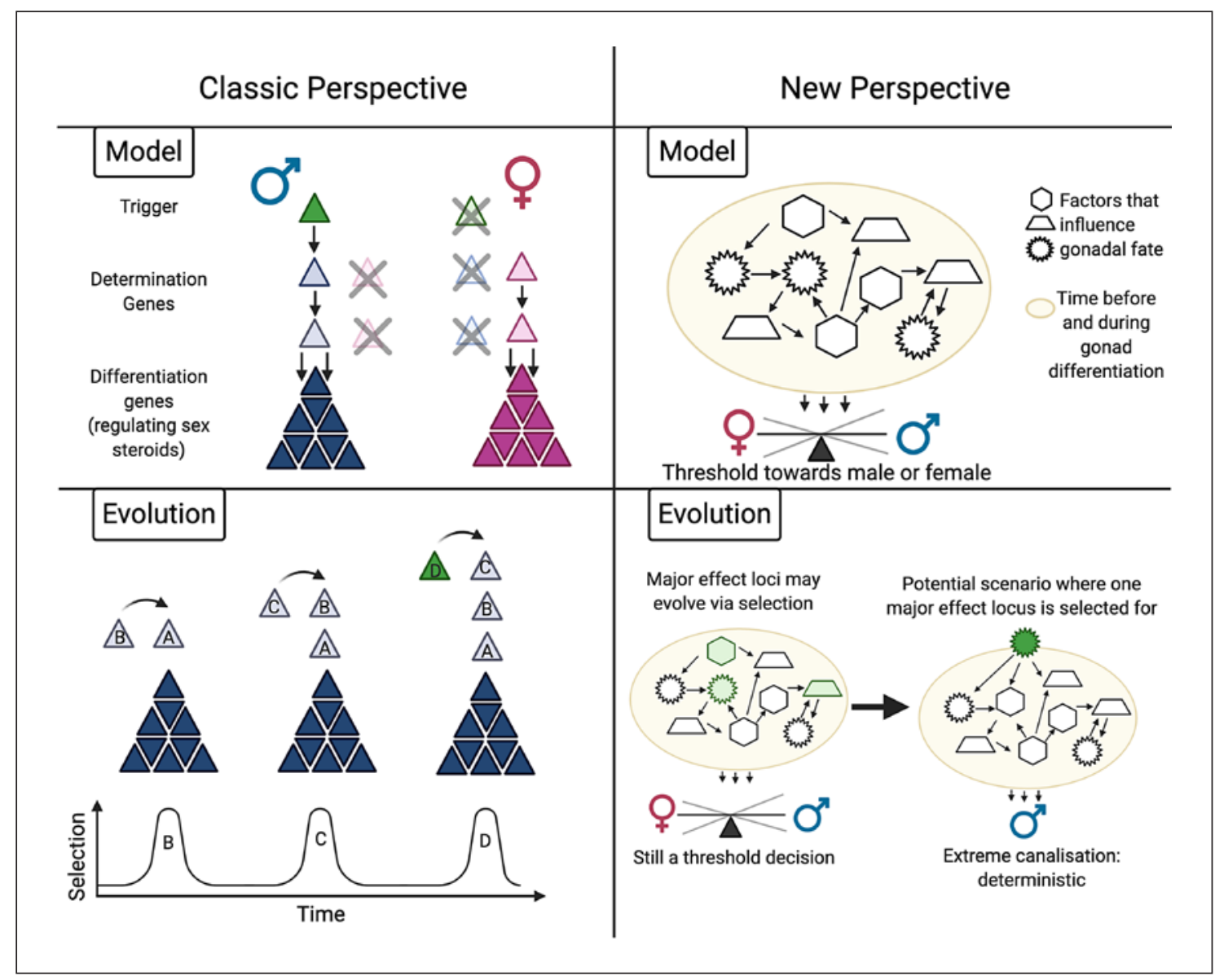

Fig. 3. Perspectives on the evolution of sex determination pathways. The classic perspective [Wilkins, 1995, 2005] and new perspective [Crews and Bull, 2009; Uller and Helanterä, 2011; Heule et al., 2014] of sex determination and sex determination evolution following each model. Adapted from Heule et al. [2014]. Created with BioRender.com.

11-ketotestosterone which is the primary androgen in fish and thus inductive of male fate [Devlin and Nagahama, 2002]. These enzymes are upregulated by $a m h$ (anti-müllerian hormone) expression [Todd et al., 2016]. Anti-müllerian hormone also acts to repress cyp19a1a alongside $d m r t 1$ and sox9 [Todd et al., 2016]. It is apparent that these 2 competing pathways both use transcriptional regulation to influence steroidogenesis as an endpoint effector - with both pathways repressing the opposing pathway [Herpin and Schartl, 2011, 2015].

\section{Paradigms of Sex Determination}

As stated earlier, the classic sex pathway model has a trigger that leads to a sexual fate decision and subsequent sexual differentiation. Wilkins [1995, 2005] suggested that such pathways might evolve from the bottom up on the basis that the downstream effectors show greater conservation than the upstream regulators. His hypothesis suggests that steps in the developmental cascade evolved in retrograde, with effectors emerging first followed by subsequent regulators leading to the initial trigger, with selection favouring each step for establishment [Wilkins, 1995, 2005] (Fig. 3). As such, sexual differentiation and effector genes are the evolutionarily most ancient ones, followed by sex determination genes, and then the initial trigger (genic or otherwise) as the most recent portion of the pathway. The addition of genes to the top of the pathway ends when a gene switch becomes fixed in the evolution of sex chromosomes [Wilkins, 1995, 2005], just as $S R Y$ is fixed on the Y chromosome across most mammals. This classical model can account for sex changes where the initial trigger is environmental; however, we contend 
that this model is largely based off a dichotomous view, perhaps limited by our cultural expectations of sex that have historically been of the view that sex is a binary phenotype that is fixed for life during development.

Emerging models favour sex as a reaction norm [AhKing and Nylin, 2010], where the phenotype is influenced by the genotype and environmental exposures, and they incorporate selection so that variation is included in the framework and not handled as an anomaly [Ah-King and Nylin, 2010]. These models and the plasticity that they allow align well with sequentially hermaphroditic fish, which change sex in response to social or ecological cues: As the external exposures change, gene expression changes result in an altered phenotype. An intermediate model to the view of Wilkins $[1995,2005]$ and the reaction norm of Ah-King and Nylin [2010] is presented by Heule et al. [2014]. This model moves away from the classical view of the sex-determining cascade towards a sex determination framework where male and female states are threshold phenotypes [Crews and Bull, 2009; Uller and Helanterä, 2011]. They propose that the process of sex determination and differentiation should be seen as a modulated network which incorporates many factors and pathways towards a phenotypic threshold. In this model, it occurs in extreme canalisation instances that a master sex determination gene may arise (Fig. 3).

\section{Models for Gene Evolution and Functional Diversity}

Given the significant variability in the sex determination and differentiation pathways in fish, particularly among master regulators [Matsuda et al., 2002; Hattori et al., 2012; Kamiya et al., 2012; Myosho et al., 2012; Yano et al., 2012; Li et al., 2015], it seems clear that understanding sex change in fish necessitates an understanding of how new genes emerge and how they are seconded into new roles. De novo formation, where genes evolve from nongenic DNA sequence [McLysaght and Guerzoni, 2015; Van Oss and Carvunis, 2019], seems to be a likely source of novelty, but actual instances of de novo gene formation are difficult to verify. De novo gene formation is not as well-characterised as other mechanisms such as gene duplication [Van Oss and Carvunis, 2019], which is a wellknown substrate for divergent gene evolution [Ohno, 1966, 1970a; Hughes, 1994; Conant and Wolfe, 2008], albeit loss of function (non-functionalization) through mutation or deletion is the most common fate of duplicated genes [Jaillon et al., 2004; Brunet et al., 2006] leading to pseudogenisation and ultimately decay.

Gene Duplication, Neofunctionalization, and the Sexual Plasticity of Fish

\section{Potential of Teleost Genomic Architecture to Contribute to Sexual Plasticity}

Genomic equivalence suggests that all cells have the potential to develop into any specific cell type. Similarly, sexually labile fish have the same capacity to be of either sex (as they can change functional sex) and even in gonochorists sex reversal can be induced [Paul-Prasanth et al., 2013]. The bipotentiality of the primordial gonad in combination with near identical gene content across sexes, particularly in species with young homomorphic chromosomes, highlights sex as an inherently plastic phenotype [Wilkins, 1995; Ellegren and Parsch, 2007]. The sexual plasticity (including hermaphroditism) of teleost fish is unique among vertebrates. Why might teleosts be more sexually plastic than other groups of vertebrates? One unique aspect that may allow for this plasticity is that teleosts underwent a WGD event 320-350 million years ago [Glasauer and Neuhauss, 2014]. Multiple lines of evidence support this WGD event, such as the duplication and synteny of the highly conserved Hox genes across teleosts [Crow et al., 2006], even in basal groups [Henkel et al., 2012], and whole-genome sequencing studies [Jaillon et al., 2004]. The WGD event was followed by subsequent rediploidisation in most teleosts, yet genomic evidence of ancestral duplication will be present in the form of retained duplicate genes [Glasauer and Neuhauss, 2014].

In comparison to their non-sex-changing counterparts, might it be possible that rediploidisation might be rare or less complete in many of the fish species that can change sex? Such a possibility has yet to be thoroughly and quantitatively explored, but if rediploidisation is indeed uncommon or more incomplete among sex-changing fish, in comparison to their gonochoristic counterparts, the resulting excess of gene duplicates would provide them with a broad array of genes to facilitate such impressive flexibility in sex. Such a finding is not without precedent, with an over-retention in regulatory genes post-WGD previously described in bony fishes [Brunet et al., 2006]. The main benefit of WGD acts through gene dosage balance where duplicates are selected for on a network level so as not to disrupt dosage-specific regulation. Gene dosage balance results in the capacity for network evolution to occur (Fig. 4), which could be a mechanism that may help to promote sexual plasticity in teleosts.

As noted earlier, the fact that hermaphroditism has arisen independently multiple times, makes it unlikely that a single factor explains all occurrences of natural sex change in fishes. WGD in teleosts, in and of itself, is not 


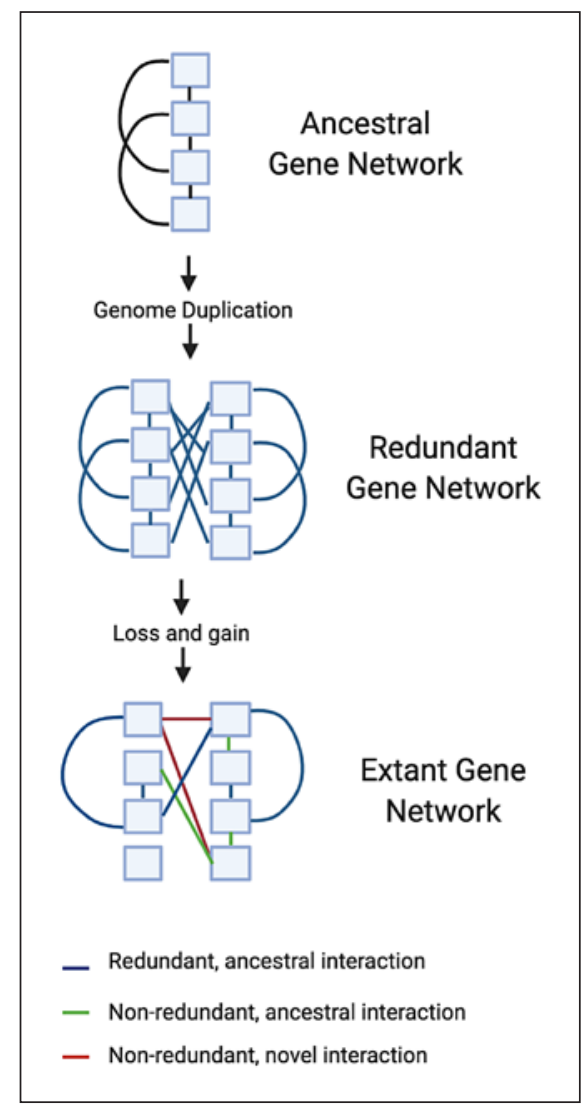

Fig. 4. Network evolution after whole-genome duplication. Squares denote gene products, interactions are denoted by lines. Adapted from Conant and Wolfe [2008]. Created with BioRender.com.

an adequate explanation of the prevalence of sex change across the teleosts. The occurrence of hermaphroditism, intersex individuals, and high degrees of sexual plasticity in other taxa, such as lamprey [Docker et al., 2019], a member of the Agnatha that predate the WGD event, also runs contrary to expectations if indeed WGD was the sole explanation proposed for the sexual plasticity of teleosts. But WGD may provide some of the capacity for teleosts to manifest some of their sexual plasticity.

Gene duplication can also occur on smaller scales. Duplication of chromosomes or smaller DNA regions may result in a novel function but may be selected against due to genetic dosage imbalances and dysregulation may be selected against (or for) as the regulatory mechanism may not have been duplicated with it [Ohno, 1970b]. Single gene duplications are widely known to effect sex determination, differentiation, and sex reversal in many animal systems and have been extensively reviewed [Herpin and Schartl, 2015; Capel, 2017).
While it requires further investigation, we posit that WGD and subsequent incomplete rediploidisation, or the favoured halting of rediploidisation of key genes, in combination with small-scale duplication due to selection favouring sex change may underpin the plasticity observed in teleost fish due to the opportunity for increased diversity to arise from existing networks through neofunctionalization and subfunctionalization. With the rapid rise in the availability of whole-genome data for many vertebrates, including fishes, this idea may now be amiable to systematic investigation.

\section{Neofunctionalization}

Neofunctionalization occurs after duplication, when one of the gene duplicates gains a function that was not present in the original gene and is selected for [Conant and Wolfe, 2008]. It is one way that diverse function can come into fruition. Ohno [1970a] put forth that mutations in one of the duplicates can give rise to a new version of a gene, because with 2 functional copies there is redundancy. Such redundancy means that the duplicated copy is free from selection, allowing the copy the freedom to nonfunctionalize (previously restricted due to purifying selection) before neofunctionalizing, after which the new function can be established in the population through positive selection [Ohno, 1970a].

\section{Subfunctionalization}

Subfunctionalization is when the original function of the ancestral gene is shared between the 2 duplicates, with neither duplicate able to carry out the entire ancestral function [Conant and Wolfe, 2008]. Hughes [1994] suggests that genes with apparent "new" function may have gained this via subfunctionalization whereby pleiotropic ancestral genes have undergone duplication, allowing for diversification and subsequent specialisation so that each function of the original gene is able to be independently optimised. Duplication frees the genes from asymmetrical purifying selection and allows positive selection for the optimisation of all functions; the escape from adaptive conflict model. Another model of subfunctionalization is the duplication, degeneration, complementation model (DDC) of Force et al. [1999], whereby both copies of the gene undergo mutation, but each retains a different aspect of the original function, i.e., the 2 copies are complementary so retention of duplicates must be selected for [Force et al., 1999]. 


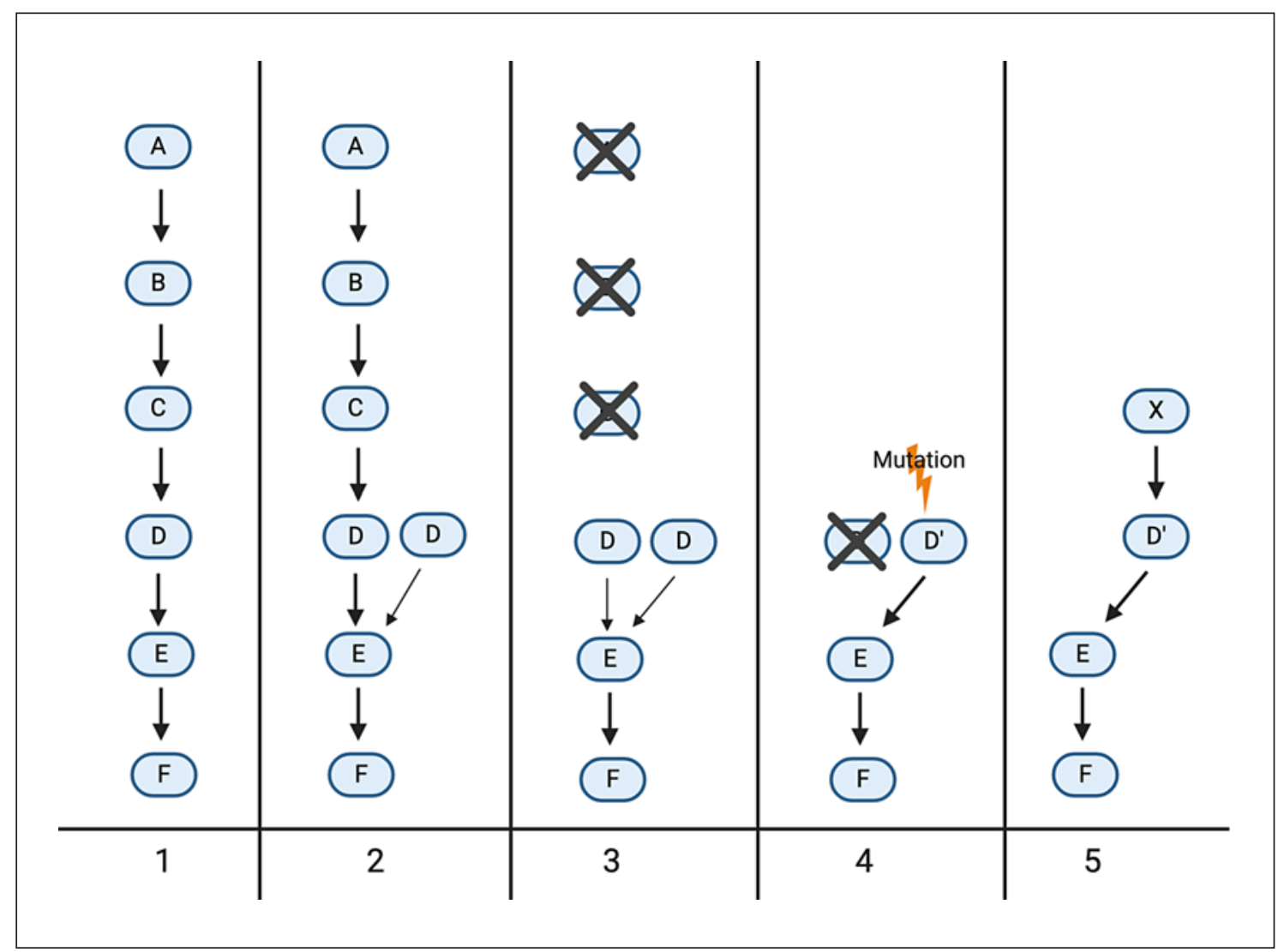

Fig. 5. How gene duplication could contribute to the evolution of the sex-determining cascade. 1 Sex determination cascade. 2 Gene duplication of D, with basal levels of transcription. 3 The basal levels of the 2 copies of D are sufficient for E; upstream components redundant. 4 Mutation of one copy allows for the basal transcription of $\mathrm{D}^{\prime}$ to be sufficient to activate E. $5 \mathrm{~A}$ new gene may be added to become a new master regulator. Arrows represent activation, thickness represents degree of contributive activation. Figure adapted from Schartl [2004]. Created with BioRender.com.

\section{Isoforms}

Gene isoforms are mRNAs with different sequence, untranslated regions or transcription start sites but originate from the same locus. Even within one single gene copy, a diversity of functional transcripts and proteins can be created through mechanisms such as alternative splicing, which can result in different isoforms, each with unique function [Xing and Lee, 2006]. However, as stated, these transcripts would map back to the same genomic locus/gene. Gene isoforms can be investigated with techniques such as RNA-Seq, and it is important to distinguish between isoforms and gene duplicates when investigating new function.

\section{Duplicated Genes Are Common in Sex Determination and Differentiation Pathways}

Many genes in the sex-change pathway of different telost fish have derived from duplication events. Often, a duplicated downstream effector gene has gained new function to become the sex-determining gene. $d m y$ is the sex-determining gene in O. latipes [Matsuda et al., 2002] and originated from a duplicate copy of autosomal $d m r t 1$ which itself is critical in the male developmental pathway across vertebrates. In Nile tilapia $a m h \Delta-y$ contains a deletion and was previously described as amhy by a group who suggested it to be the sex-determining locus in a Swansea strain of Nile tilapia based on a linkage mapping approach [Eshel et al., 2014]. However, Li et al. [2015] found $a m h \Delta-y$ to be an allelic, Y-specific duplicate to the 
amh gene (located on the X-chromosome). They found that it is in fact a tandem duplicate of $a m h \Delta-y$ with a missense SNP termed amhy that is the sex-determining gene in Nile tilapia [Li et al., 2015] (or at least the strain they investigated). These duplications of sex pathway genes and subsequent evolution into sex determination genes makes sense in light of Schartl's model [Schartl, 2004] where a hierarchical mode can be disrupted through duplication and dosage imbalance, followed by mutation, enabling the duplicate to be sufficient for sex determination (Fig. 5).

Interestingly, of the 6 master-regulators known in fish, only one is not derived from a gene duplicated from within the sexual development cascade. In rainbow trout $s d Y$ is thought to have evolved from an immune-related gene [Yano et al., 2012]. It is hypothesized to have arisen through duplication and subsequent neofunctionalization, but high sequence divergence prevents accurately elucidating its evolutionary history [Yano et al., 2012].

The 2 aromatase genes of teleosts, cyp 19a1a and cyp$19 a 1 b$, likely arose and were retained from the WGD event. These 2 genes are a good example of subfunctionalization as they have diverged to have different spatial expression; cyp19a1a is expressed gonadally, whereas $c y$ p19a1b is expressed primarily in the brain [Chiang et al., 2001; Page et al., 2010; Zhang et al., 2014]. Gonadal aromatase (cyp19a1a) is required for ovarian differentiation and must be actively repressed for testicular differentiation to proceed [Guiguen et al., 2010] and shows femalebiased expression in multiple transcriptomic studies [Manousaki et al., 2014; Liu et al., 2015; Todd et al., 2019]. However, even though these genes are considered among the most conserved female-specific genes across vertebrates, one study investigating 5 species of cichlid fish found that cyp19a1 a had gained a new expression pattern; being expressed in the testis in the ectodine lineage [Böhne et al., 2013]. Additionally, cyp19a1b was highly expressed in the testis of the Haplochromini group [Böhne et al., 2013]. This not only indicates that functional divergence from a duplication event provides flexibility, but also demonstrates that variation is not only present at the "top" of sex-determination cascades but also at the effector level - leaning into the proposed paradigm shift away from master-effector sex determination towards a network-level integration model [Heule et al., 2014].

The androgen receptor genes $a r-a$ and $a r-b$ also arose through the teleost WGD event. However, not all teleosts retained the duplication. Basal teleosts and percomorphs retained the 2 functional copies, in percom- orphs the 2 androgen receptors had diverged from each other more than they had in the basal species [Douard et al., 2008]. There was sequence divergence specific to the percomorph lineage which is implicated in functional difference. Further, Douard et al. [2008] found that the pattern of substitutions between $a r-a$ and $a r-b$ differed significantly between each other suggesting the 2 receptors have experienced different selection pressures. The authors label this as putative neofunctionalization until tested in vivo and note that it is tempting to link this to the sexual plasticity of percomorphs as a group [Douard et al., 2008]. Ogino et al. [2016] concurred with the timing of the ar duplication event and went further to demonstrate functional differences in medaka, documenting the different expression regions relevant to the gonad and demonstrating that $a r-a$ has an amino acid change that causes hyperactivity, altering its nuclear localisation to achieve constitutive and increasing transactivation. Although the biological implications of this increase are still to be investigated, this is an apparent example of neofunctionalization in androgen receptors resulting from an ancient duplication event. A comparison of paralogues in tetraodon to human orthologues has shown that some sets of genes are retained after duplication with bias towards biological processes such as development; however, these results were limited by small sample size [Brunet et al., 2006]. While this study was undertaken in a gonochorist, this bias in retention of developmental genes in combination with the bias in retention of regulatory genes [Brunet et al., 2006] could be enriched and contribute further to the sexual plasticity observed in teleost fish.

Wingless-type MMTV integration site family member 4 (wnt4) is a gene implicated in ovarian development and shows female-biased expression in many species [Tsakogiannis et al., 2019]. It has presumably been duplicated through the teleost WGD and retained in at least 2 copies in some lineages. In black porgy (a protandrous sequential hermaphrodite), there are 15 copies of wnt4 [Zhang et al., 2018] which is in stark comparison to the 2 copies in zebrafish and 3 copies in rainbow trout [Zhang et al., 2018]. However, wnt 4 is not differentially expressed in Diplodus puntazzo (a rudimentary hermaphrodite) although $B$-catenin, which is involved in the same pathway, is differentially expressed [Manousaki et al., 2014]. Of particular interest are the wnt4 paralogues in bluehead wrasse. In a transcriptomic analysis of protogynous sex change, Todd et al. [2019] found that wnt4a was downregulated as sex change progressed from female to male, a pattern that was expected based on studies of oth- 
er vertebrates. However the paralogue, $w n t 4 b$, was upregulated and demonstrated testis-specific expression [Todd et al., 2019], a pattern opposite to its assumed function, given wnt 4 is one of the key antagonistic players in feminisation. This expression pattern suggests that the duplicated $w n t 4$ has been neofunctionalized, gaining a testis-specific role in bluehead wrasse. Further research into this commonly duplicated gene in other hermaphroditic species is warranted. Overall, several lines of evidence indicate that duplication and subsequent retention and evolution of sex pathway genes may be a contributing factor to the remarkable sexual plasticity of teleost fish.

Just as for cell type canalisation, epigenetics plays a role in sex pathway antagonism [Ortega-Recalde et al., 2020]. Epigenetic mechanisms can incorporate environmental exposures and consequently differentially up- or downregulate genes through changes in chromatin structure, histone modification, and CpG methylation which can affect the availability of genes for transcription and the recruitment of transcriptional machinery [Ortega-Recalde et al., 2020]. Of note, methylation of CpG islands is associated with the silencing of genes [Ortega-Recalde et al., 2020], likely due to steric hindrance. As such, the epigenetic machinery may be able to facilitate the switch between antagonistic pathways. Among those genes that appear to be epigenetically controlled is aromatase, $c y$ p19a1a, which becomes increasingly heavily methylated during sex change in bluehead wrasse [Todd et al., 2019].

The establishment and maintenance of DNA methylation which results in heritable but reversible gene silencing, is the responsibility of the DNA methyltransferase genes dnmt1 (which ensures that methylation is maintained at DNA replication) [Law and Jacobsen, 2010] and $d n m t 3$ (which is essential for de novo methylation) [Chen et al., 2003]. These $d n m t$ genes are also duplicated in teleosts as a result of the WGD event. Additionally, there are lineage-specific duplications of $d n m t$ genes; for example, $d n m t 3$ has 6 paralogues in zebrafish [Campos et al., 2012]. Transcriptomic profiling of the bluehead wrasse found that dnmt3 genes had varying female and male expression bias potentially implicating them in sex change [Liu et al., 2015; Todd et al., 2019]. Recent comparative transcriptomic analysis has revealed further evidence of sex-biased gonadal gene expression of epigenetic regulators among 5 species of sparid fishes, including species-specific differences across reproductive modes (rudimentary hermaphroditism, protogyny, protandry, and gonochorism) [Tsakogiannis et al., 2019]. Transcriptomic analyses indicated that $d n m t$ gene expression altered significantly as protogynous sex change progressed, female-specific dnmt genes were downregulated, while male-specific dnmt genes were upregulated [Tsakogiannis et al., 2019]. The role of these genes, in combination with their sex-specific expression and duplication in some lineages indicates that epigenetic regulation could be integral to the sexual plasticity that is observed in teleost fish [Ortega-Recalde et al., 2020].

\section{Conclusion}

While we now understand the evolutionary advantages of sex change, and the structural and hormonal changes that accompany this transformation, we are only beginning to comprehend the genetic cascades that orchestrate the sex-change process and how they have evolved. Gene duplication provides the raw material for divergence, variation, and freedom of evolution at the molecular level. Sex determination networks are bistable, antagonistic, and require ongoing maintenance. Such maintenance is disrupted in sequentially hermaphroditic fish, and the genetic triggers that flip the switch, or elements that alter the threshold towards alternative sexual fate, are still under investigation. Gene duplication and neofunctionalization are implicated in generating diversity within sex pathways, including novel master regulators and diverse patterns of sex-specific expression. That neofunctionalization of duplicate sex-pathway genes has played a key role in the evolution of the sexual plasticity in teleosts is a hypothesis that warrants further investigation.

Recent genomic advances and the increasing quantity and quality of fish genomic resources creates the opportunity for in-depth investigations of the genome-scale processes that may have provided the foundational framework for the sexual flexibility we see in the teleosts. Over the coming years, the role of gene duplication and neofunctionalization in the sexual plasticity of fish will no doubt be investigated, providing new insights into how sex change in fish has evolved, and contribute to our overall understanding of developmental pathway evolution and sex determination across the vertebrates.

\section{Conflict of Interest Statement}

The authors have no conflicts of interest to declare. 


\section{Funding Sources}

This study was supported by the University of Otago (J.E., L.U., N.J.G.), Deakin University (E.V.T.), and Alexander von Humbolt Feodor Lynen Research Fellowship (L.U.).

\section{Author Contributions}

N.J.G. conceived the review, and J.E. wrote the first draft with editorial input from N.J.G., L.U., and E.V.T. All authors approved the final version.

\section{References}

Adolfi MC, Nakajima RT, Nóbrega RH, Schartl M. Intersex, hermaphroditism, and gonadal plasticity in vertebrates: Evolution of the Müllerian duct and $\mathrm{Amh} / \mathrm{Amhr} 2$ signaling. Annu Rev Anim Biosci. 2019;7:149-72.

Ah-King M, Nylin S. Sex in an evolutionary perspective: Just another reaction norm. Evol Biol. 2010;37(4):234-46.

Bachtrog D, Mank JE, Peichel CL, Kirkpatrick M, Otto SP, Ashman TL, et al. Sex determination: Why so many ways of doing it?. PLoS Biol. 2014;12(7):e1001899.

Böhne A, Heule C, Boileau N, Salzburger W. Expression and sequence evolution of aromatase cyp19a1 and other sexual development genes in East African cichlid fishes. Mol Biol Evol. 2013;30(10):2268-85.

Brunet FG, Roest Crollius H, Paris M, Aury JM, Gibert P, Jaillon O, et al. Gene loss and evolutionary rates following whole-genome duplication in teleost fishes. Mol Biol Evol. 2006; 23(9):1808-16.

Campos C, Valente LM, Fernandes JM. Molecular evolution of zebrafish dnmt 3 genes and thermal plasticity of their expression during embryonic development. Gene. 2012;500(1):93100.

Capel B. Vertebrate sex determination: evolutionary plasticity of a fundamental switch. Nat Rev Genet. 2017;18(11):675-89.

Chen T, Ueda Y, Dodge JE, Wang Z, Li E. Establishment and Maintenance of Genomic Methylation Patterns in Mouse Embryonic Stem Cells by Dnmt3a and Dnmt3b. Mol Cell Biol. 2003;23(16):5594-605.

Chiang EF, Yan YL, Guiguen Y, Postlethwait J, Chung BC. Two Cyp19 (P450 Aromatase) genes on duplicated zebrafish chromosomes are expressed in ovary or brain. Mol Biol Evol. 2001;18(4):542-50.

Conant GC, Wolfe KH. Turning a hobby into a job: How duplicated genes find new functions. Nat Rev Genet. 2008;9(12):938-50.

Crews D, Bull JJ. Mode and tempo in environmental sex determination in vertebrates. Semin Cell Dev Biol. 2009;20:251-5.

Crow KD, Stadler PF, Lynch VJ, Amemiya C, Wagner GP. The "fish-specific" Hox cluster duplication is coincident with the origin of teleosts. Mol Biol Evol. 2006;23(1):121-36.

Devlin RH, Nagahama Y. Sex determination and sex differentiation in fish: an overview of genetic, physiological, and environmental influences. Aquaculture. 2002;208(3-4):191-364.
Docker MF, Beamish FWH, Yasmin T, Bryan MB, Khan A. The Lamprey gonad. In: Docker M, editor. Lampreys: Biology, Conservation and Control. Fish \& Fisheries Series, Vol 38. Dordrecht: Springer; 2019.

Douard V, Brunet F, Boussau B, Ahrens-Fath I, Vlaeminck-Guillem V, Haendler B, et al. The fate of the duplicated androgen receptor in fishes: a late neofunctionalization event? BMC Evol Biol. 2008;8:336.

Ellegren H, Parsch J. The evolution of sex-biased genes and sex-biased gene expression. Nat Rev Genet. 2007;8(9):689-98.

Eshel O, Shirak A, Dor L, Band M, Zak T, Markovich-Gordon $\mathrm{M}$, et al. Identification of male-specific Amh duplication, sexually differentially expressed genes and microRNAs at early embryonic development of Nile tilapia (Oreochromis niloticus). BMC Genomics. 2014; $15: 774$

Force A, Lynch M, Pickett FB, Amores A, Yan YL, Postlethwait J. Preservation of duplicate genes by complementary, degenerative mutations. Genetics. 1999;151(4):1531-45.

Fricke H, Fricke S. Monogamy and sex change by aggressive dominance in coral reef fish. Nature. 1977;266(5605):830-2.

Ghiselin MT. The evolution of hermaphroditism among animals. Q Rev Biol. 1969;44(2):189208.

Glasauer SM, Neuhauss SC. Whole-genome duplication in teleost fishes and its evolutionary consequences. Mol Genet Genomics. 2014; 289(6):1045-60.

Guiguen Y, Fostier A, Piferrer F, Chang CF. Ovarian aromatase and estrogens: A pivotal role for gonadal sex differentiation and sex change in fish. Gen Comp Endocrinol. 2010;165(3): 352-66.

Hattori A. High mobility of the protandrous anemonefish Amphiprion frenatus: nonrandom pair formation in limited shelter space. Ichthyol Res. 2005;52(1):57-63.

Hattori RS, Murai Y, Oura M, Masuda S, Majhi SK, Sakamoto T, et al. A Y-linked anti-Müllerian hormone duplication takes over a critical role in sex determination. Proc Natl Acad Sci USA. 2012;109(8):2955-9.

Henkel CV, Burgerhout E, de Wijze DL, Dirks RP, Minegishi Y, Jansen HJ, et al. Primitive duplicate Hox clusters in the European eel's genome. PLoS One. 2012;7(2):e32231.

Herpin A, Schartl M. Sex determination: Switch and suppress. Curr Biol. 2011;21(17):R656-9.
Herpin A, Schartl M. Plasticity of gene-regulatory networks controlling sex determination: of masters, slaves, usual suspects, newcomers, and usurpators. EMBO Rep. 2015;16:126074.

Heule C, Salzburger W, Böhne A. Genetics of sexual development: An evolutionary playground for fish. Genetics. 2014;196(3):57991.

Hodge JR, Santini F, Wainwright PC. Correlated evolution of sex allocation and mating system in wrasses and parrotfishes. Am Nat. 2020; 196(1):57-73.

Huang JD, Lee MF, Chang CF. The morphology of gonadal tissue and male germ cell in the protandrous black porgy, Acanthopagrus schlegeli. Zool Stud. 2002;41(2):216-27.

Hughes AL. The evolution of functionally novel proteins after gene duplication. Proc Biol Sci. 1994;256(1346):119-24.

Jaillon O, Aury JM, Brunet F, Petit JL, StangeThomann N, Mauceli E, et al. Genome duplication in the teleost fish Tetraodon nigroviridis reveals the early vertebrate proto-karyotype. Nature. 2004;431(7011):946-57.

Kamiya T, Kai W, Tasumi S, Oka A, Matsunaga $\mathrm{T}$, Mizuno N, et al. A trans-species missense $\mathrm{SNP}$ in Amhr2 is associated with sex determination in the Tiger Pufferfish, Takifugu rubripes (Fugu). PLoS Genet. 2012;8(7): e1002798.

Kobayashi Y, Sunobe T, Kobayashi T, Nagahama Y, Nakamura M. Gonadal structure of the serial-sex changing gobiid fish Trimma okinawae. Dev Growth Differ. 2005;47(1):7-13.

Kuwamura T, Sunobe T, Sakai Y, Kadota T, Sawada K. Hermaphroditism in fishes: an annotated list of species, phylogeny, and mating system. Ichthyol Res. 2020;67(3):341-60.

Lamm MS, Liu H, Gemmell NJ, Godwin JR. The need for speed: Neuroendocrine regulation of socially-controlled sex change. Integr Comp Biol. 2015;55(2):307-22.

Law JA, Jacobsen SE. Establishing, maintaining and modifying DNA methylation patterns in plants and animals. Nat Rev Genet. 2010; 11(3):204-20.

Lee MF, Huang JD, Chang CF. The development of ovarian tissue and female germ cells in the protandrous black porgy fish, Acanthopagrus schlegeli. Zool Stud. 2008;47(3):302-16.

Li M, Sun Y, Zhao J, Shi H, Zeng S, Ye K, et al. A Tandem Duplicate of Anti-Müllerian Hormone with a Missense SNP on the Y Chromosome Is Essential for Male Sex Determination in Nile Tilapia, Oreochromis niloticus. PLoS Genet. 2015;11(11):e1005678. 
Liu H, Lamm MS, Rutherford K, Black MA, Godwin JR, Gemmell NJ. Large-scale transcriptome sequencing reveals novel expression patterns for key sex-related genes in a sexchanging fish. Biol Sex Differ. 2015;6:26.

Mank JE, Promislow DEL, Avise JC. Evolution of alternative sex-determining mechanisms in teleost fishes. Biol J Linn Soc. 2006;87(1):8393.

Manousaki T, Tsakogiannis A, Lagnel J, Sarropoulou E, Xiang JZ, Papandroulakis N, et al. The sex-specific transcriptome of the hermaphrodite sparid sharpsnout seabream (Diplodus puntazzo). BMC Genomics. 2014;15: 655-16.

Matsuda M: Genetic Control of Sex Determination and Differentiation in Fish. In: Kobayashi K, Kitano T, Iwao Y, Kondo M, editors. Reproductive and Developmental Strategies. Diversity and Commonality in Animals. Tokyo: Springer; 2018.

Matsuda M, Nagahama Y, Shinomiya A, Sato T, Matsuda C, Kobayashi T, et al. DMY is a Yspecific DM-domain gene required for male development in the medaka fish. Nature. 2002;417(6888):559-63.

McLysaght A, Guerzoni D. New genes from noncoding sequence: the role of de novo proteincoding genes in eukaryotic evolutionary innovation. Philos Trans R Soc Lond B Biol Sci. 2015;370(1678):20140332.

Munday P. Bi-directional sex change: testing the growth-rate advantage model. Behav Ecol Sociobiol. 2002;52(3):247-54.

Munday PL, Buston PM, Warner RR. Diversity and flexibility of sex-change strategies in animals. Trends Ecol Evol (Amst). 2006;21(2): 89-95.

Myosho T, Otake H, Masuyama H, Matsuda M, Kuroki Y, Fujiyama A, et al. Tracing the emergence of a novel sex-determining gene in $\mathrm{Me}$ daka, Oryzias luzonensis. Genetics. 2012; 191(1):163-70

Nakamura M, Kobayashi Y, Miura S, Alam MA, Bhandari RK. Sex change in coral reef fish. Fish Physiol Biochem. 2005;31(2-3): 117.

Nakashima Y, Kuwamura T, Yogo Y. Both-ways sex change in monogamous coral gobies, Gobiodon spp. Environ Biol Fish. 1996;46(3): 281-8.

Ogino Y, Kuraku S, Ishibashi $\mathrm{H}$, Miyakawa $\mathrm{H}$, Sumiya E, Miyagawa S, et al. Neofunctionalization of androgen receptor by gain-of-function mutations in teleost fish lineage. Mol Biol Evol. 2016;33(1):228-44.
Ohno S. Polyphyletic Evolution of Vertebrates. In: Ohno S, editor. Sex Chromosomes and Sex-Linked Genes. Berlin, Heidelberg: Springer; 1966. p. 24-46.

Ohno S. The Creation of a New Gene from a Redundant Duplicate of an Old Gene [Internet]. In: Ohno S, editor. Evolution by Gene Duplication. Berlin, Heidelberg: Springer; 1970a. p. 71-82.

Ohno S. Tandem Duplication Involving Part of One Linkage Group at a Time [Internet]. In: Ohno S, editor. Evolution by Gene Duplication. Berlin, Heidelberg: Springer; 1970b. p. 89-97.

Ortega-Recalde O, Goikoetxea A, Hore TA, Todd EV, Gemmell NJ. The genetics and epigenetics of sex change in fish. Annu Rev Anim Biosci. 2020;8:47-69.

Page YL, Diotel N, Vaillant C, Pellegrini E, Anglade I, Mérot Y, et al. Aromatase, brain sexualization and plasticity: the fish paradigm. Eur J Neurosci. 2010;32:2105-15.

Paul-Prasanth B, Bhandari RK, Kobayashi T, Horiguchi R, Kobayashi Y, Nakamoto M, et al. Estrogen oversees the maintenance of the female genetic program in terminally differentiated gonochorists. Sci Rep. 2013;3:286211.

Policansky D. Sex change in plants and animals. Annu Rev Ecol Syst. 1982;13(1):471-95.

Reinboth R. Physiological problems of teleost ambisexuality. Environ Biol Fish. 1988;22(4): 249-59.

Schartl M. Sex chromosome evolution in nonmammalian vertebrates. Curr Opin Genet Dev. 2004;14(6):634-41.

Takahashi H. Juvenile Hermaphroditism in the Zebrafish, Brachydanio rerio. Bull Fac Fish Hokkaido Univ. 1977;28:57-65.

Todd EV, Liu H, Muncaster S, Gemmell NJ. Bending genders: The biology of natural sex change in fish. Sex Dev. 2016;10(5-6):223-41.

Todd EV, Ortega-Recalde O, Liu H, Lamm MS, Rutherford KM, Cross H, et al. Stress, novel sex genes, and epigenetic reprogramming orchestrate socially controlled sex change. Sci Adv. 2019;5(7):eaaw7006.

Tsakogiannis A, Manousaki T, Lagnel J, Papanikolaou N, Papandroulakis N, Mylonas CC, et al. The gene toolkit implicated in functional sex in sparidae hermaphrodites: inferences from comparative transcriptomics. Front Genet. 2019;9:749.
Uller T, Helanterä H. From the origin of sex-determining factors to the evolution of sex-determining systems. Q Rev Biol. 2011;86(3): 163-80.

Van Oss SB, Carvunis AR. De novo gene birth. PLoS Genet. 2019;15(5):e1008160.

Warner RR. The adaptive significance of sequential hermaphroditism in animals. Am Nat. 1975;109(965):61-82.

Warner RR. Sex change and the size-advantage model. Trends Ecol Evol (Amst). 1988;3(6): 133-6.

Warner RR, Swearer SE. Social control of sex change in the bluehead wrasse, Thalassoma bifasciatum (Pisces: Labridae). Biol Bull. 1991;181(2):199-204.

Wilkins AS. Moving up the hierarchy: A hypothesis on the evolution of a genetic sex determination pathway. BioEssays. 1995; 17(1):71-7.

Wilkins AS. Recasting developmental evolution in terms of genetic pathway and network evolution ... and the implications for comparative biology. Brain Res Bull. 2005;66(4-6): 495-509.

Wu GC, Tomy S, Lee MF, Lee YH, Yueh WS, Lin $\mathrm{CJ}$, et al. Sex differentiation and sex change in the protandrous black porgy, Acanthopagrus schlegeli. Gen Comp Endocrinol. 2010; 167(3):417-21.

Xing Y, Lee C. Alternative splicing and RNA selection pressure--evolutionary consequences for eukaryotic genomes. Nat Rev Genet. 2006; 7(7):499-509.

Yano A, Guyomard R, Nicol B, Jouanno E, Quillet E, Klopp C, et al. An immune-related gene evolved into the master sex-determining gene in Rainbow Trout, Oncorhynchus mykiss. Curr Biol. 2012;22(15):1423-8.

Zhang K, Xu J, Zhang Z, Huang Y, Ruan Z, Chen $S$, et al. A comparative transcriptomic study on developmental gonads provides novel insights into sex change in the protandrous black porgy (Acanthopagrus schlegelii). Genomics. 2019;111(3):277-83.

Zhang Y, Zhang S, Lu H, Zhang L, Zhang W. Genes encoding aromatases in teleosts: Evolution and expression regulation. Gen Comp Endocrinol. 2014;205:151-8.

Zhang Z, Zhang K, Chen S, Zhang Z, Zhang J, You $\mathrm{X}$, et al. Draft genome of the protandrous Chinese black porgy, Acanthopagrus schlegelii. Gigascience. 2018;7(4):1. 